\title{
Pyronaridine-Artesunate versus Mefloquine plus Artesunate for Malaria
}

\author{
Ronnatrai Rueangweerayut, M.D., Aung Pyae Phyo, M.D., Chirapong Uthaisin, M.D., \\ Yi Poravuth, M.D., Tran Quang Binh, M.D., Ph.D., Halidou Tinto, Ph.D., \\ Louis K. Pénali, Ph.D., Neena Valecha, M.D., Nong Thi Tien, M.D., Ph.D., \\ Salim Abdulla, M.D., Ph.D., Isabelle Borghini-Fuhrer, Ph.D., Stephan Duparc, M.D., \\ Chang-Sik Shin, Ph.D., and Lawrence Fleckenstein, Pharm.D., \\ for the Pyronaridine-Artesunate Study Team*
}

ABSTRACT

From the Department of Internal Medicine, Mae Sot General Hospital (R.R.) Shoklo Malaria Research Unit, Mae Sot (A.P.P.), and Mae Ramat Hospital (C.U.) - all in Tak, Thailand; the National Malaria Center, Phnom Penh, Cambodia (Y.P.); Dak O Health Center, Choray Hospital, Ho Chi Minh City (T.Q.B.), and the Commune Xy Health Center, National Institute of Malariology, Parasitology and Entomology, Hanoi (N.T.T.) - both in Vietnam; West African Network II for Monitoring Antimalarial Treatment, Center Muraz, Bobo Dioulasso, Burkina Faso (H.T.); Institut Pasteur, Abidjan, Ivory Coast (L.K.P.); National Institute of Malaria Research, Delhi, India (N.V.); Ifakara Health Institute, Dar es Salaam, Tanzania (S.A.); Medicines for Malaria Venture, Geneva (I.B.-F., S.D.); Shin Poong Pharmaceutical Company, Seoul, South Korea (C.-S.S.); and the College of Pharmacy, University of lowa, lowa City (L.F.). Address reprint requests to Dr. BorghiniFuhrer at Medicines for Malaria Venture, International Center Cointrin, Rte. de PréBois 20, P.O. Box 1826, CH-1215 Geneva 15, Switzerland, or at borghinii@mmv.org.

*Additional members of the PyronaridineArtesunate Study Team are listed in the Supplementary Appendix, available at NEJM.org.

N Engl J Med 2012;366:1298-309. Copyright @ 2012 Massachusetts Medical Society.

\section{BACKGROUND}

Pyronaridine-artesunate is an artemisinin-based combination therapy under evaluation for the treatment of Plasmodium falciparum and P. vivax malaria.

\section{METHODS}

We conducted a phase 3, open-label, multicenter, noninferiority trial that included 1271 patients between 3 and 60 years of age from Asia (81.3\%) or Africa (18.7\%) with microscopically confirmed, uncomplicated $P$. falciparum malaria. Patients underwent randomization for treatment with a fixed-dose combination of $180 \mathrm{mg}$ of pyronaridine and $60 \mathrm{mg}$ of artesunate or with $250 \mathrm{mg}$ of mefloquine plus $100 \mathrm{mg}$ of artesunate. Doses were calculated according to body weight and administered once daily for 3 days.

\section{RESULTS}

Pyronaridine-artesunate was noninferior to mefloquine plus artesunate for the primary outcome: adequate clinical and parasitologic response in the per-protocol population on day 28 , corrected for reinfection with the use of polymerase-chain-reaction (PCR) genotyping. For this outcome, efficacy in the group receiving pyronaridineartesunate was $99.2 \%$ (743 of 749 patients; $95 \%$ confidence interval [CI], 98.3 to 99.7) and that in the group receiving mefloquine plus artesunate was $97.8 \%$ (360 of 368 patients; $95 \%$ CI, 95.8 to 99.1), with a treatment difference of 1.4 percentage points (95\% CI, 0.0 to 3.5; $\mathrm{P}=0.05$ ). In the intention-to-treat population, efficacy on day 42 in the group receiving pyronaridine-artesunate was $83.1 \%$ (705 of 848 patients; $95 \%$ CI, 80.4 to 85.6) and that in the group receiving mefloquine plus artesunate was $83.9 \%$ (355 of 423 patients; $95 \%$ CI, 80.1 to 87.3). In Cambodia, where there were 211 study patients, the median parasite clearance time was prolonged for both treatments: 64 hours versus 16.0 to 38.9 hours in other countries ( $\mathrm{P}<0.001$, on the basis of Kaplan-Meier estimates). Kaplan-Meier estimates of the recrudescence rate in the intention-to-treat population in Cambodia until day 42 were higher with pyronaridine-artesunate than with mefloquine plus artesunate $(10.2 \%$ [ $95 \% \mathrm{CI}, 5.4$ to 18.6$]$ vs. $0 \% ; \mathrm{P}=0.04$ as calculated with the log-rank test), but similar for the other countries combined (4.7\% [95\% CI, 3.3 to 6.7] and $2.8 \%$ [ $95 \% \mathrm{CI}, 1.5$ to 5.3], respectively; $\mathrm{P}=0.24$ ). Elevated levels of aminotransferases were observed in those receiving pyronaridine-artesunate. Two patients receiving mefloquine plus artesunate had seizures.

\section{CONCLUSIONS}

Fixed-dose pyronaridine-artesunate was efficacious in the treatment of uncomplicated P. falciparum malaria. In Cambodia, extended parasite clearance times were suggestive of in vivo resistance to artemisinin. (Funded by Shin Poong Pharmaceutical Company and the Medicines for Malaria Venture; ClinicalTrials.gov number, NCT00403260.) 
RTEMISININ-BASED COMBINATION THERapy is critical for the effective treatment and control of Plasmodium falciparum malaria. ${ }^{1-4}$ However, reports from the Cambodian-Thai border indicate the emergence of artemisinin tolerance or resistance in P. falciparum. ${ }^{2-8}$

Pyronaridine-artesunate is a fixed-dose, artemisinin-based combination therapy that is being developed for the treatment of uncomplicated $P$. falciparum and P. vivax malaria. ${ }^{9-11}$ In phase 2 and 3 clinical trials, a fixed-dose, 3:1 ratio of pyronaridine to artesunate has shown high efficacy in the treatment of falciparum malaria, with cure rates on day 28 of more than $98 \%$ (corrected for reinfection with the use of polymerase-chainreaction [PCR] genotyping). ${ }^{9,10}$ In a phase 3 study conducted in Africa and Asia, the reported efficacy of pyronaridine-artesunate among Asian patients was 98.1\% (102 of 104 patients) and was equivalent to that of artemether-lumefantrine. ${ }^{10}$ In the treatment of patients with P. vivax malaria, the efficacy of pyronaridine-artesunate was similar to that of chloroquine, but with faster clearance times for parasites $(\mathrm{P}<0.001)$ and for fever $(\mathrm{P}=0.002){ }^{11}$

This phase 3 trial compared the efficacy and safety of a fixed-dose combination of pyronaridine-artesunate in Asian and African patients with acute, uncomplicated P. falciparum monoinfection with that of a loose combination of mefloquine plus artesunate. The primary objective was to test the noninferiority of pyronaridine-artesunate to mefloquine plus artesunate for efficacy on the basis of adequate clinical and parasitologic response on day 28, corrected for reinfection with PCR genotyping. ${ }^{12}$ Given the importance of the continued efficacy of artemisinin-based combination therapy, we also looked for evidence of artemisinin resistance by examining data on parasite clearance time.

\section{METHODS}

\section{STUDY DESIGN}

The study was conducted from January 25, 2007, until October 2, 2008, at local hospitals and health centers in Mae Sot and Mae Ramat, Thailand; Pailin, Cambodia; Ho Chi Minh City and Hanoi, Vietnam; Bobo Dioulasso, Burkina Faso; Abidjan, Ivory Coast; Bagamoyo, Tanzania; and Mangalore, India. The institutional review board at each participating center approved the protocol, which was implemented in accordance with the provisions of the Declaration of Helsinki and Good Clinical
Practice guidelines and with local laws and regulations. (The protocol is available with the full text of this article at NEJM.org.) All participants or their parents or guardians provided written informed consent; assent was required from children who were able to understand the study.

The study was designed by the authors and the study sponsors, the Medicines for Malaria Venture and Shin Poong Pharmaceutical Company. All the authors vouch for the completeness and accuracy of the data and the analysis and for the fidelity of the study to the protocol. The pyronaridine-artesunate used in the study (a tablet with $180 \mathrm{mg}$ of pyronaridine and $60 \mathrm{mg}$ of artesunate) was donated by Shin Poong Pharmaceutical Company. Mefloquine tablets (250 mg) and artesunate tablets (100 mg) were purchased by the study sponsors from Mepha. Averion International (now part of Aptiv Solutions) provided the computer-generated randomization schedule, and Magenta Communications developed the first draft of the manuscript from a study report approved by the authors and also coordinated the authors' contributions.

We assumed that with an adequate clinical and parasitologic response rate to both treatments of $93 \%$ and a noninferiority limit of $5 \%$, the acquisition of data on 1140 evaluable subjects (with 2 patients receiving pyronaridine-artesunate for every 1 receiving mefloquine plus artesunate [760:380]) would provide $90 \%$ power to demonstrate the noninferiority of pyronaridine-artesunate when using a two-sided $95 \%$ confidence interval. A drop-out rate of $10 \%$ was assumed, resulting in a target for recruitment of 1269 subjects (with 846 receiving pyronaridine-artesunate and 423 mefloquine plus artesunate).

Randomization numbers were assigned in ascending order according to center. Drugs were administered by an investigator who was aware of group assignments; clinical and parasitologic assessments were performed by investigators who were not aware of group assignments.

\section{PATIENTS}

Eligible subjects were of either sex, were 3 to 60 years of age, and weighed between 20 and $90 \mathrm{~kg}$ (without severe malnutrition), and they presented with microscopically confirmed P. falciparum monoinfection (1000 to 100,000 asexual parasites per cubic millimeter) and fever that had developed within the preceding 24 hours. Exclusion criteria were complicated or severe malaria, ${ }^{13}$ anemia (characterized as a hemoglobin level 
$<8$ g per deciliter), severe vomiting, diarrhea, pregnancy or lactation, any clinically significant illness other than malaria, hepatic or renal impairment, known hypersensitivity or allergy to the study drugs, use of an antimalarial drug in the preceding 2 weeks, use of an investigational drug in the preceding 4 weeks, and previous participation in this study.

\section{TREATMENT AND PROCEDURES}

Study treatments were administered orally with water once daily for 3 days (days 0,1 , and 2). The target dosage range for pyronaridine-artesunate was 7.2:2.4 mg per kilogram of body weight to 13.8:4.6 mg per kilogram; the actual doses were 7.1 to $14.0 \mathrm{mg}$ per kilogram for pyronaridine and 2.4 to $4.7 \mathrm{mg}$ per kilogram for artesunate. The target range for mefloquine plus artesunate was 5.6:2.2 mg per kilogram to $12.5: 5.0 \mathrm{mg}$ per kilogram; the actual doses were 6.2 to $12.5 \mathrm{mg}$ per kilogram for mefloquine and 2.5 to $5.0 \mathrm{mg}$ per kilogram for artesunate. Patients who vomited within 30 minutes of taking the first dose were given a repeat dose. Vomiting of subsequent doses led to study withdrawal and administration of rescue medication. Patients remained hospitalized during treatment, with follow-up at days 7, 14, 21, 28,35 , and 42 .

\section{PARASITOLOGIC ANALYSIS}

Parasite densities were determined in accordance with methods published by the World Health Organization (WHO). ${ }^{12}$ Duplicate thick blood smears were used to establish mean parasite counts. The smears were examined independently by two microscopists at screening (to determine study eligibility), on day 0 (before dosing), and every 8 hours thereafter until 72 hours or more had passed or until two consecutive negative readings were recorded at least 7 and up to 25 hours apart, at the end of therapy, at all follow-up visits, and in the event of treatment failure.

Thin blood smears were taken at screening, on day 0 (before dosing), and from day 7 onward whenever parasitologic blood samples were taken. PCR genotyping was performed with the use of a standard protocol at a central laboratory (Swiss Tropical and Public Health Institute, Basel). Recrudescence was defined as the detection of at least one matching allelic band in the highly polymorphic genes encoding the surface proteins of the P. falciparum merozoite (MSP1 and MSP2) and glutamate-rich protein (GLURP). ${ }^{14,15}$ This analysis was performed with samples obtained at baseline as compared with those obtained after day 7 .

\section{OUTCOMES}

Efficacy outcomes were determined on the basis of WHO definitions. ${ }^{12}$ The primary outcome was adequate clinical and parasitologic response (i.e., aparasitemia, regardless of axillary temperature and without evidence of previous treatment failure) on day 28 , corrected for reinfection with the use of PCR genotyping. Secondary end points were adequate clinical and parasitologic response without correction for reinfection, parasite clearance time (time from first dose to asexual aparasitemia, determined on the basis of two consecutive negative findings recorded at least 7 and up to 25 hours apart), fever clearance time (time from first dose to apyrexia as determined by two consecutive normal temperature readings recorded at least 7 and up to 25 hours apart), and the proportions of patients who were aparasitic or apyretic on days 1, 2, and 3. Exploratory end points included adequate clinical and parasitologic response on day 42 and gametocyte clearance time. Safety outcomes were based on the incidence of adverse events, the results of urinalysis and other clinical laboratory tests, and the results of electrocardiography.

\section{STATISTICAL ANALYSIS}

The intention-to-treat population included all patients who underwent randomization and received at least one treatment dose and was equivalent to the safety population. The per-protocol population included all patients who underwent randomization, completed a full treatment course or had early treatment failure before treatment was completed, had outcome data for the primary efficacy end point on day 28 , and complied with the protocol to the extent that would allow efficacy evaluation.

The primary efficacy analysis was evaluated for the per-protocol population. The noninferiority of pyronaridine-artesunate to mefloquine plus artesunate was assumed if the lower limit of the twosided $95 \%$ confidence interval (calculated with the Newcombe-Wilson method) for the difference in adequate clinical and parasitologic response, corrected for reinfection, was not lower than $-5 \%$. If pyronaridine-artesunate was noninferior, superiority $(\mathrm{P}<0.05)$ was tested with the use of a twosided chi-square test. The exact two-sided 95\% confidence interval (calculated with Pearson-Clopper limits) for the primary end point was also 
calculated. Similar analyses were conducted for uncorrected adequate clinical and parasitologic response, outcomes on day 42 , and the intentionto-treat population.

Post hoc Kaplan-Meier analyses with a log-rank test were used to compare rates of recrudescence and reinfection. Data were censored for patients without the event (recrudescence or reinfection) at the last available date for parasite assessment. In the case of major deviations from the protocol, data for patients without the event were censored at the last parasite assessment before the protocol violation. In the intention-to-treat population, Kaplan-Meier estimates were also used to analyze parasite, fever, and gametocyte clearance times. (The plan for statistical analysis is available in the protocol.)

\section{RESULTS}

\section{PATIENTS}

The intention-to-treat population included $1271 \mathrm{pa}-$ tients; 848 received pyronaridine-artesunate, and 423 received mefloquine plus artesunate (Fig. 1). Baseline characteristics in the two treatment groups were similar (see Table 1 in the Supplementary Appendix, available at NEJM.org). Patients from Asian centers constituted 1033 of the 1271 patients (81.3\%) (Table 2 in the Supplementary Appendix).

\section{EFFICACY}

Pyronaridine-artesunate was noninferior to mefloquine plus artesunate for the primary outcome: adequate clinical and parasitologic response in the per-protocol population on day 28 , corrected for reinfection with the use of PCR genotyping. For this outcome, efficacy in the group receiving pyronaridine-artesunate was $99.2 \%$ (743 of 749 patients; $95 \%$ confidence interval [CI], 98.3 to 99.7) and that in the group receiving mefloquine plus artesunate was $97.8 \%$ (360 of 368 patients; $95 \%$ CI, 95.8 to 99.1), with a treatment difference of 1.4 percentage points ( $95 \% \mathrm{CI}, 0.0$ to $3.5 ; \mathrm{P}=0.05$ ). Among patients 12 years of age or younger who received pyronaridine-artesunate, the efficacy rate was $99.1 \%$ (111 of 112 patients; 95\% CI, 95.1 to 100), which was similar to the rate for patients older than 12 years of age (99.2\% [632 of 637 patients]; 95\% CI, 98.2 to 99.7). In the group receiving mefloquine plus artesunate, efficacy was also similar among patients 12 years of age or younger and among patients older than 12 years of age, with efficacy rates of $100 \%$ (63 of 63 patients; $95 \%$ CI, 94.3 to
100) and $97.4 \%$ (297 of 305 patients; 95\% CI, 94.9 to 98.9), respectively. The corrected efficacy rate on day 28 in the per-protocol population was at least $96.6 \%$ across all centers for both study treatments except in Mae Ramat, Thailand, where the efficacy rate in the group receiving mefloquine plus artesunate was $94.7 \%$ (89 of 94 patients; $95 \%$ CI, 88.0 to 98.3 ) (Table 4 in the Supplementary Appendix).

In the intention-to-treat population, adequate clinical and parasitologic response on day 42, corrected for reinfection, was $83.1 \%$ (705 of 848 patients; $95 \% \mathrm{CI}, 80.4$ to 85.6 ) in the pyronaridine-artesunate group and $83.9 \%$ (355 of 423 patients; $95 \% \mathrm{CI}, 80.1$ to 87.3 ) in the mefloquineplus-artesunate group. Results for the intentionto-treat population are shown in Table 1.

Kaplan-Meier estimates of the recrudescence rate in the intention-to-treat population through day 42 were $5.3 \%$ (95\% CI, 3.9 to 7.3) in the pyronaridine-artesunate group and 2.5\% (95\% CI, 1.3 to 4.7) in the mefloquine-plus-artesunate group. The log-rank test indicated that the recrudescence rate was slightly higher with pyronaridine-artesunate than with mefloquine plus artesunate through day $42(\mathrm{P}=0.049)$ (Fig. 2A). Reinfection rates were similar for the two treatments: $9.2 \%$ (95\% CI, 7.3 to 11.6) and 6.2 (95\% CI, 4.1 to 9.2), respectively $(\mathrm{P}=0.17)$ (Fig. 2B). The rate of P. falciparum reappearance (recrudescence or reinfection) (Fig. 2C) was higher among patients in the pyronaridineartesunate group (14.2\%; 95\% CI, 11.9 to 17.0 ) than it was among those in the mefloquine-plusartesunate group $(8.6 \%$; $95 \% \mathrm{CI}, 6.1$ to 11.9$)$ $(\mathrm{P}=0.02)$. Kaplan-Meier estimates of postbaseline P. vivax infection (Fig. 2D) were higher in the pyronaridine-artesunate group $(11.1 \%$; $95 \% \mathrm{CI}$, 9.0 to 13.7) than in the group receiving mefloquine plus artesunate $(1.8 \%$; $95 \% \mathrm{CI}, 0.8$ to 3.9$)$ through day $42(\mathrm{P}<0.001)$.

Median parasite clearance time was approximately 32 hours in both groups, with at least $92 \%$ of patients free of parasitemia by day 3 (Table 5 in the Supplementary Appendix). Kaplan-Meier analysis indicated that there were no significant differences between treatment groups in parasite clearance time $(\mathrm{P}=0.08)$, fever clearance time $(\mathrm{P}=0.60)$, or gametocyte clearance time $(\mathrm{P}=0.10)$.

\section{ARTEMISININ RESISTANCE IN CAMBODIA}

Among the 211 patients in Pailin, Cambodia, parasite clearance time was significantly extended in both treatment groups, whether it was compared 


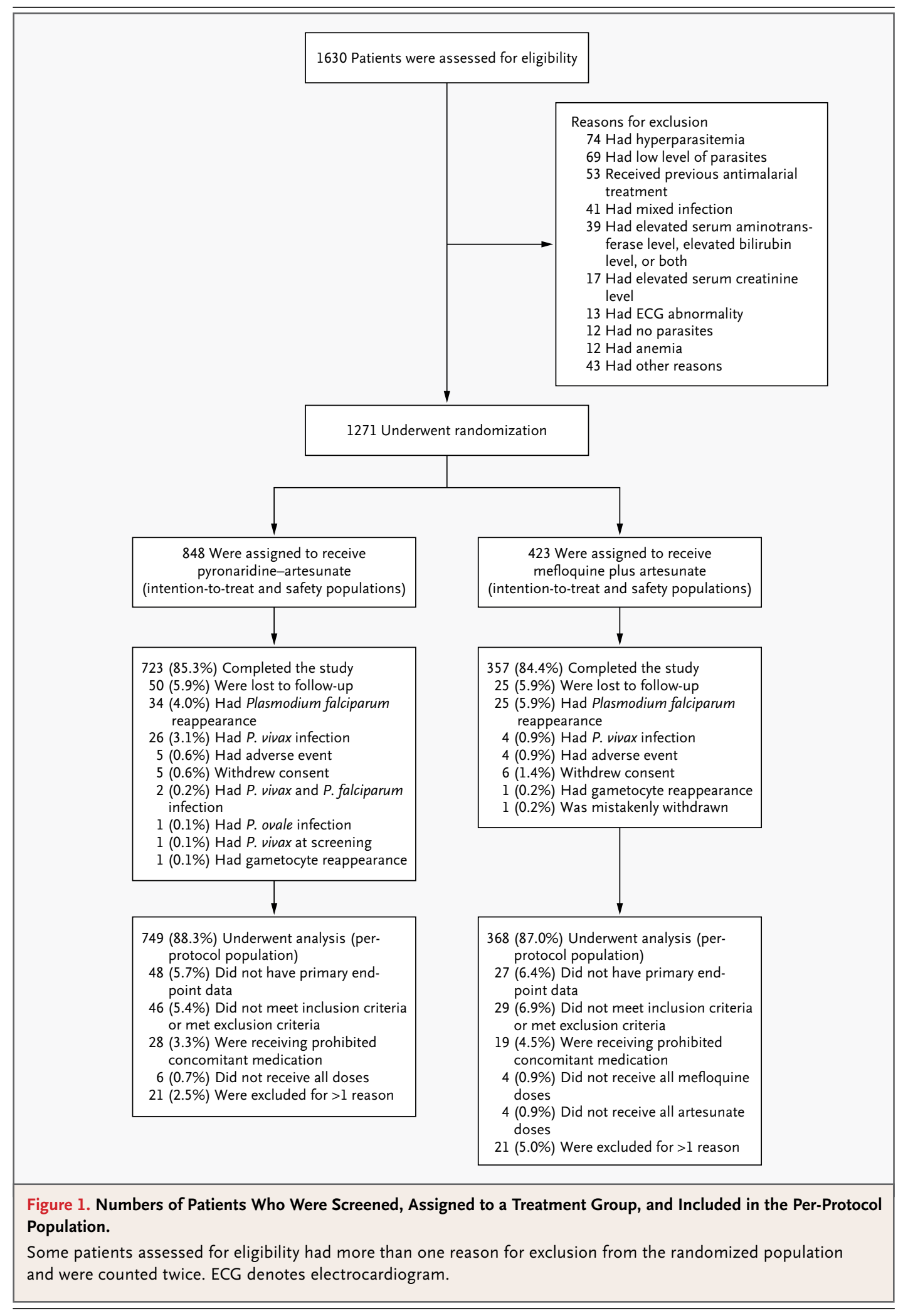




\begin{tabular}{|c|c|c|c|c|}
\hline Outcome & $\begin{array}{l}\text { Pyronaridine-Artesunate } \\
\qquad(\mathrm{N}=848)\end{array}$ & $\begin{array}{l}\text { Mefloquine plus Artesunate } \\
\qquad(\mathrm{N}=423)\end{array}$ & Difference $(95 \% \mathrm{Cl})$ & P Value \\
\hline \multicolumn{5}{|l|}{ ACPR, Day 28} \\
\hline \multicolumn{5}{|c|}{ Corrected for reinfection with PCR genotyping* } \\
\hline No. of patients & 793 & 387 & & \\
\hline$\%$ of patients $(95 \% \mathrm{Cl})$ & 93.5 (91.6 to 95.1$)$ & 91.5 (88.4 to 94.0$)$ & $2.0(-0.9$ to 5.4$)$ & 0.19 \\
\hline No. with missing data (\%) $\dagger$ & $48(5.7)$ & $27(6.4)$ & & \\
\hline No. with early treatment failure (\%) & 0 & $1(0.2)$ & & \\
\hline No. with late clinical failure (\%) & 0 & 0 & & \\
\hline No. with late parasitologic failure (\%) & $7(0.8)$ & $8(1.9)$ & & \\
\hline Total no. of failures (\%) & $55(6.5)$ & $36(8.5)$ & & \\
\hline \multicolumn{5}{|l|}{ Not corrected for reinfection } \\
\hline No. of patients & 789 & 382 & & \\
\hline$\%$ of patients $(95 \% \mathrm{Cl})$ & 93.0 (91.1 to 94.7$)$ & $90.3(87.1$ to 93.0$)$ & $2.7(-0.4$ to 6.3$)$ & 0.09 \\
\hline No. with missing data (\%) $\dagger$ & $47(5.5)$ & $24(5.7)$ & & \\
\hline No. with early treatment failure (\%) & 0 & $1(0.2)$ & & \\
\hline No. with late clinical failure (\%) & $2(0.2)$ & 0 & & \\
\hline No. with late parasitologic failure (\%) & $10(1.2)$ & $16(3.8)$ & & \\
\hline Total no. of failures (\%) & $59(7.0)$ & $41(9.7)$ & & \\
\hline \multicolumn{5}{|l|}{ ACPR, Day 42} \\
\hline \multicolumn{5}{|l|}{ Corrected for reinfection with PCR genotyping* } \\
\hline No. of patients & 705 & 355 & & \\
\hline$\%$ of patients $(95 \% \mathrm{Cl})$ & 83.1 (80.4 to 85.6$)$ & $83.9(80.1$ to 87.3$)$ & $-0.8(-4.9$ to 3.7$)$ & 0.72 \\
\hline No. with missing data (\%) $†$ & $99(11.7)$ & $55(13.0)$ & & \\
\hline No. with early treatment failure (\%) & 0 & $1(0.2)$ & & \\
\hline No. with late clinical failure (\%) & $5(0.6)$ & 0 & & \\
\hline No. with late parasitologic failure (\%) & $39(4.6)$ & $12(2.8)$ & & \\
\hline Total no. of failures (\%) & $143(16.9)$ & $68(16.1)$ & & \\
\hline \multicolumn{5}{|l|}{ Not corrected for reinfection } \\
\hline No. of patients & 708 & 351 & & \\
\hline$\%$ of patients $(95 \% \mathrm{Cl})$ & 83.5 (80.8 to 85.9$)$ & $83.0(79.1$ to 86.4$)$ & $0.5(-3.7$ to 5.0$)$ & 0.82 \\
\hline No. with missing data (\%) $†$ & $85(10.0)$ & $40(9.5)$ & & \\
\hline No. with early treatment failure (\%) & 0 & $1(0.2)$ & & \\
\hline No. with late clinical failure (\%) & $14(1.7)$ & $1(0.2)$ & & \\
\hline No. with late parasitologic failure (\%) & $41(4.8)$ & $30(7.1)$ & & \\
\hline Total no. of failures (\%) & $140(16.5)$ & $72(17.0)$ & & \\
\hline
\end{tabular}

* On day 28, there were no cases in which correction for reinfection, performed with the use of PCR (polymerase chain reaction) genotyping, was indeterminate; there was one such case on day 42, and it was considered to be a recrudescence for the calculation of the failure rate. $\uparrow$ Patients with missing data were considered to have had treatment failure in the intention-to-treat population.

with the clearance time in other study centers individually $(\mathrm{P}<0.001)$ or when the results from these centers were combined $(\mathrm{P}<0.001)$ (Fig. $3 \mathrm{~A})$. Across both treatment groups, the median para- site clearance time was 64.1 to 64.2 hours in Cambodia versus 16.0 to 38.9 hours at all the other centers (Table 6 in the Supplementary Appendix). In Cambodia, $62.5 \%$ of patients were 


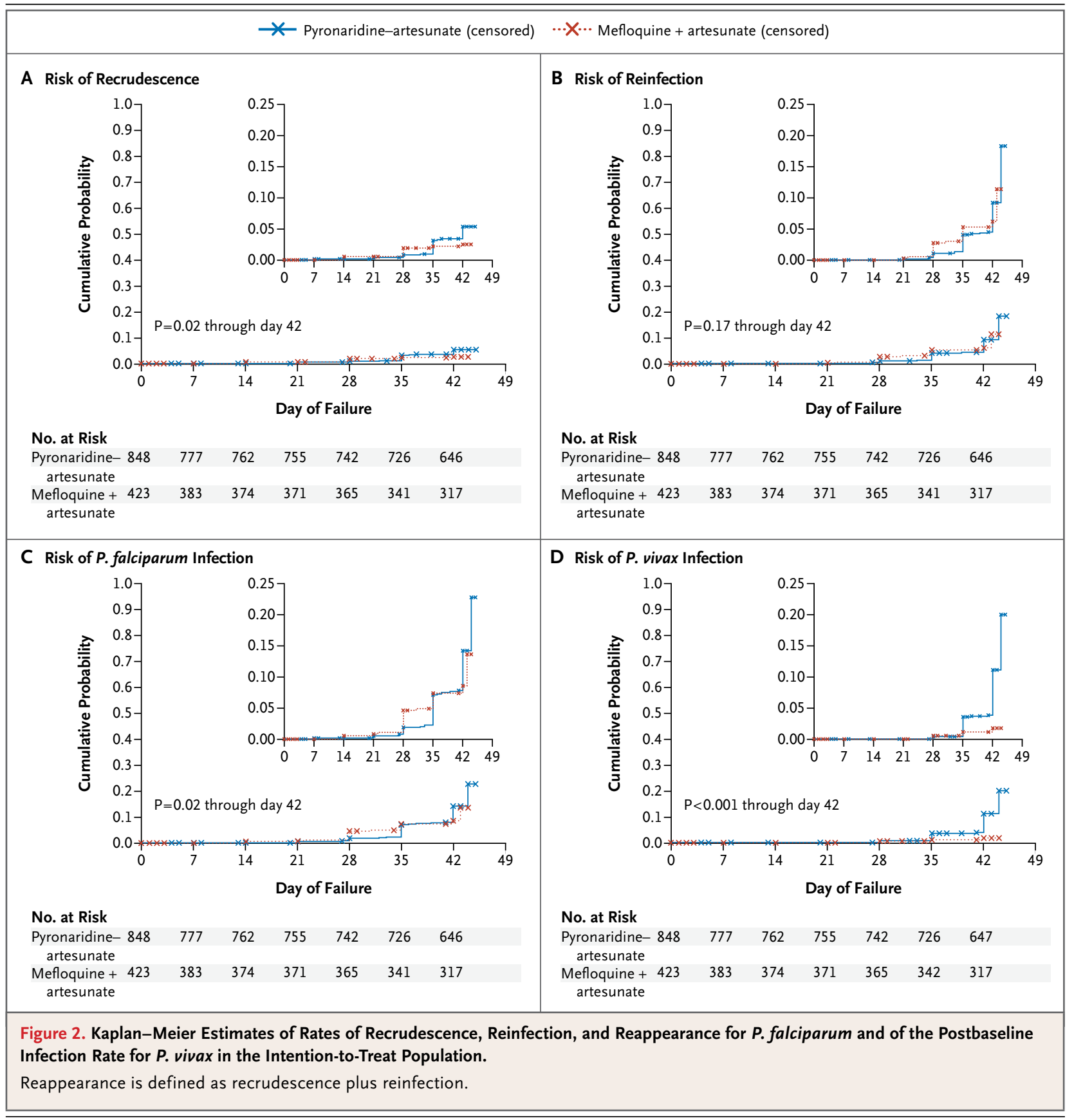

aparasitic within 72 hours of treatment initiation as compared with $98.0 \%$ of all other patients (Table 7 in the Supplementary Appendix). The effect of country on parasite clearance time remained significant $(\mathrm{P}<0.001)$ after adjustment for baseline factors (country, sex, body-mass index, and parasitemia), according to a Cox regression model.
Kaplan-Meier estimates of the recrudescence rate in Cambodia until day 42 (Fig. 3B) were higher in the group receiving pyronaridine-artesunate than in the group receiving mefloquine plus artesunate (10.2\% [95\% CI, 5.4 to 18.6] vs. $0 \% ; \mathrm{P}=0.04)$. The combined data for countries other than Cambodia showed no significant dif- 


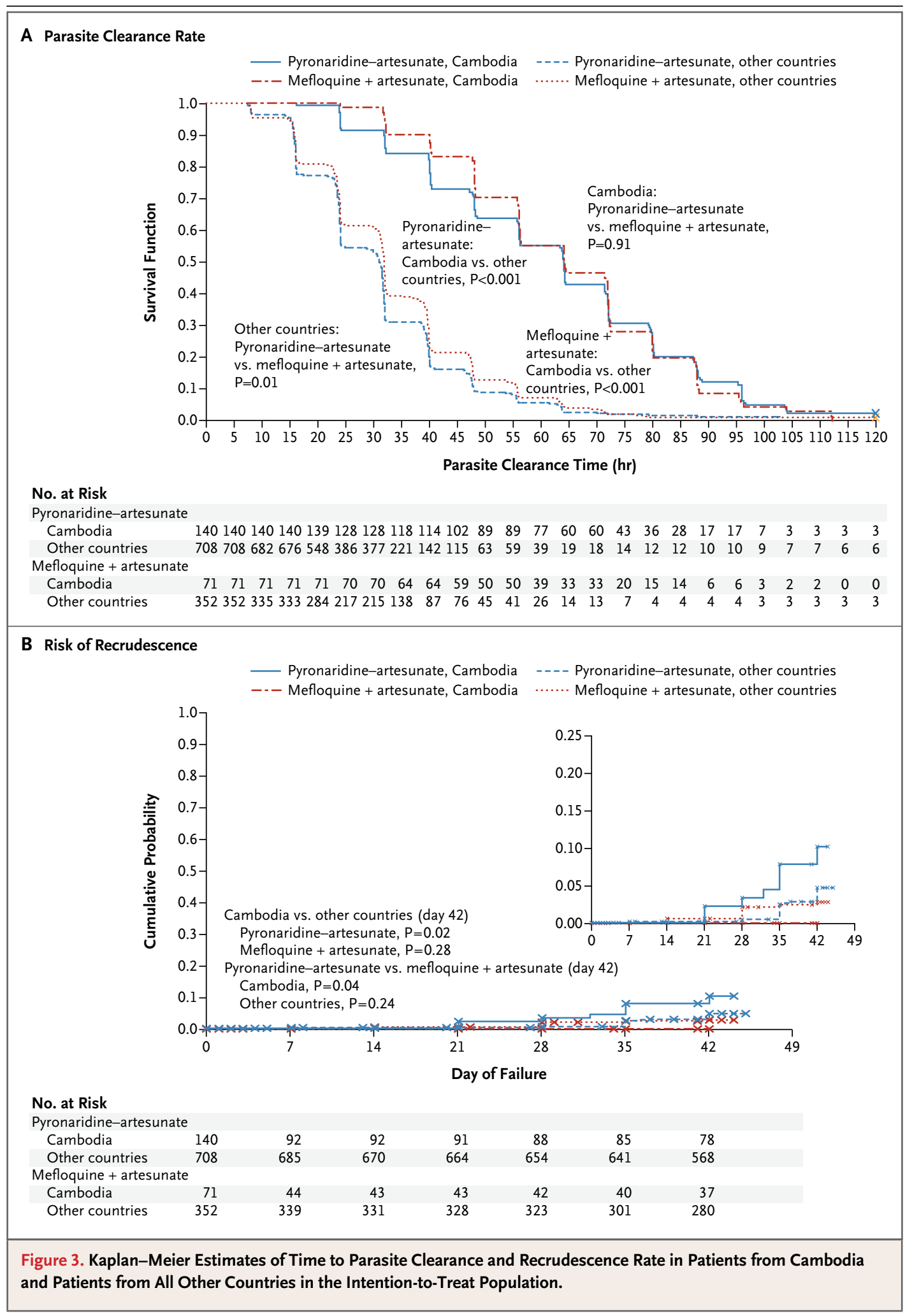


ference in recrudescence rates through day 42 between the group receiving pyronaridine-artesunate (4.7\%; 95\% CI, 3.3 to 6.7 ) and the group receiving mefloquine plus artesunate (2.8\%; 95\% CI, 1.5 to 5.3; $\mathrm{P}=0.24$ ).

There was no significant difference in exposure to pyronaridine (concentration on day 7 , or area under the concentration time curve) or to the artesunate active metabolite, dihydroartemisinin (area under the concentration time curve), in Cambodian patients as compared with those from other countries (Table 8 in the Supplementary Appendix).

\section{SAFETY}

The proportion of patients who had at least one adverse event was $45.9 \%$ for the group receiving pyronaridine-artesunate and $44.9 \%$ for the group receiving mefloquine plus artesunate (Table 2 , and Table 9 in the Supplementary Appendix). Adverse events that occurred in more than $5 \%$ of patients were headache and myalgia with pyronaridineartesunate and headache and dizziness with mefloquine plus artesunate.

There were no deaths during the study. In the safety population, there were seven serious adverse events in six patients $(0.7 \%)$ receiving pyronaridine-artesunate and three serious adverse events in three patients $(0.7 \%)$ receiving mefloquine plus artesunate (Table 2). Two of these three events (seizure and grand mal seizure) were considered to be related to treatment with mefloquine plus artesunate. Patient discontinuation of the study drug and withdrawal from the study resulted from six adverse events in five patients $(0.6 \%)$ receiving pyronaridine-artesunate (four had vomiting, and one had both nausea and vomiting) and from four adverse events in four patients $(0.9 \%)$ receiving mefloquine plus artesunate (three had vomiting, and one had cerebral malaria) (Table 2).

During the study, there were no clinically significant changes in hematologic values and no significant differences between treatment groups. In the pyronaridine-artesunate group, there were 15 patients with alanine aminotransferase levels that were more than five times the upper limit of the normal range. Among these 15 patients, 2 adult men had peak levels of alanine aminotransferase that were at least three times the upper limit of the normal range and peak bilirubin levels that were at least two times the upper limit of the normal range. However, in one of these patients, the alanine aminotransferase level was two times the upper limit of the normal range at baseline and the total bilirubin level on day 7 was the same as that at baseline (there was a decrease vs. baseline on day 3). An additional patient had levels of alanine aminotransferase and aspartate aminotransferase that were at least three times the upper limit of the normal range that first appeared on day 42. None of the patients showed any other signs or symptoms of liver disorders. Other biochemistry values in the two treatment groups were similar. Key laboratory values are shown in Tables 10 and 11 in the Supplementary Appendix.

Six patients $(0.7 \%)$ in the pyronaridine-artesunate group had abnormal electrocardiograms, as did three patients $(0.7 \%)$ in the mefloquine-plusartesunate group. All abnormalities were mild and resolved before study completion. Of those patients, one $(0.1 \%)$ in the group receiving pyronaridine-artesunate had QT prolongation, and three $(0.7 \%)$ in the group receiving mefloquine plus artesunate had a long or prolonged QT interval. None had a QT interval that exceeded $480 \mathrm{msec}$.

\section{DISCUSSION}

The WHO criterion for introducing an alternative artemisinin-based combination therapy — an adequate clinical and parasitologic response in more than $95 \%$ of patients at follow-up on day 28 - was met by pyronaridine-artesunate in all the countries in our study. ${ }^{1}$ Efficacy was generally consistent with that reported in previous studies of the use of pyronaridine-artesunate against $P$. falciparum malaria., ${ }^{9}, 10$ The recrudescence rate in the group receiving pyronaridine-artesunate was higher than that reported in an earlier phase 3 trial $-4.7 \%$ ( $95 \%$ CI, 3.3 to 6.7 ) versus $1.2 \%$ (95\% CI, 0.6 to 2.2). ${ }^{10}$ This difference can probably be attributed to the greater immunity to $P$. falciparum among the patients included in the previous trial, most of whom were recruited from African study sites where P. falciparum transmission rates are higher than those in the mostly Asian sites included in the current study. However, differences in parasite drug sensitivity cannot be discounted, because susceptibility testing was not performed in our study or the previous study. A specific pediatric formulation of pyronaridine-artesunate granules is currently under development. ${ }^{9}$ Consequently, it was not the aim of this study to determine efficacy in children, and our patient population included few 
children younger than 12 years of age. Most of the data on pyronaridine-artesunate reported so far are for immunologically mature patients..$^{9-11}$

Kaplan-Meier analysis of the intention-totreat population indicated that until day 28 , the risk of recrudescence was higher with mefloquine plus artesunate. However, through day 42, the risk of recrudescence was higher with pyronaridine-artesunate, an outcome driven by data from the Cambodian center. When data for the non-Cambodian centers were combined, there was no difference between treatment groups in the recrudescence rate through day 42 .

The greater recrudescence rate among Cambodian patients through day 42 could not be explained by insufficient exposure to pyronaridine, artesunate, or dihydroartemisinin relative to exposure among patients from other countries. Similarly, population pharmacokinetic modeling of data from this study and from previous phase 2 and phase 3 studies of pyronaridine-artesunate conducted in Cambodia, Thailand, and Tanzania showed no evidence of pharmacokinetic differences between Cambodian patients and patients from other countries with regard to pyronaridine, artesunate, or dihydroartemisinin. However, there was a trend toward slightly lower median concentrations of pyronaridine on day 7 in 23 patients with recrudescence as compared with 212 patients without recrudescence, when data from Cambodia, Thailand, and Tanzania were pooled. This trend was also evident in Cambodia, in 12 patients who had recrudescence and 88 patients who did not.

Parasite clearance time was significantly extended in Cambodian patients as compared with those in the other countries. This finding is consistent with those in other reports and indicates the presence of resistance to artemisinin. ${ }^{3,4,6,16-18}$ In artemisinin-based combination therapy, the half-life of artemisinin is much shorter than that of the partner drug, so late recrudescence should be caused by the lack of efficacy in the partner drug. ${ }^{19}$ Pyronaridine has been used only sparsely in Southeast Asia, so widespread resistance is thought to be unlikely. Also, given that its halflife is 13.2 days, pyronaridine resistance should have an effect on recrudescence by day 28 , but this was not seen. However, artemisinin resistance could have indirectly affected late outcomes. If the parasiticidal effect of artemisinin is attenuated by pharmacodynamic resistance, there is greater dependence on the patient's immune sys-
Table 2. Summary of Adverse Events.

\begin{tabular}{|c|c|c|}
\hline Event & $\begin{array}{c}\text { Pyronaridine- } \\
\text { Artesunate } \\
(\mathrm{N}=848)\end{array}$ & $\begin{array}{l}\text { Mefloquine plus } \\
\text { Artesunate } \\
(\mathrm{N}=423)\end{array}$ \\
\hline Adverse event from any cause - no. (\%)* & $389(45.9)$ & $190(44.9)$ \\
\hline Anemia & $31(3.7)$ & $17(4.0)$ \\
\hline Basophilia & $8(0.9)$ & $9(2.1)$ \\
\hline Eosinophilia & $20(2.4)$ & $5(1.2)$ \\
\hline Diarrhea & $10(1.2)$ & $9(2.1)$ \\
\hline Vomiting & $18(2.1)$ & $9(2.1)$ \\
\hline Nasopharyngitis & $21(2.5)$ & $11(2.6)$ \\
\hline $\begin{array}{l}\text { Increased alanine aminotransferase } \\
\text { level }\end{array}$ & $21(2.5)$ & 0 \\
\hline $\begin{array}{l}\text { Increased aspartate aminotransferase } \\
\text { level }\end{array}$ & $19(2.2)$ & $1(0.2)$ \\
\hline Decreased hemoglobin level & $21(2.5)$ & $7(1.7)$ \\
\hline Increased aminotransferase level & $18(2.1)$ & $3(0.7)$ \\
\hline Myalgia & $53(6.3)$ & $19(4.5)$ \\
\hline Dizziness & $26(3.1)$ & $28(6.6)$ \\
\hline Headache & $101(11.9)$ & $44(10.4)$ \\
\hline Cough & $30(3.5)$ & $10(2.4)$ \\
\hline $\begin{array}{l}\text { Serious adverse event from any cause - } \\
\text { no. (\%) }\end{array}$ & $6(0.7)$ & $3(0.7)$ \\
\hline Autoimmune hemolytic anemia & $1(0.1)$ & 0 \\
\hline Cerebral malaria & 0 & $1(0.2)$ \\
\hline Cholera & $1(0.1)$ & 0 \\
\hline Pneumonia & $1(0.1)$ & 0 \\
\hline Acute pyelonephritis & $1(0.1)$ & 0 \\
\hline Wound infection & $1(0.1)$ & 0 \\
\hline Seizure†' & 0 & $1(0.2)$ \\
\hline Grand mal seizure $†$ & 0 & $1(0.2)$ \\
\hline Abortion & $1(0.1)$ & 0 \\
\hline Depression & $1(0.1)$ & 0 \\
\hline $\begin{array}{l}\text { Adverse events resulting in discontinua- } \\
\text { tion of treatment }- \text { no. }(\%) \div\end{array}$ & $5(0.6)$ & $4(0.9)$ \\
\hline Nausea & $1(0.1)$ & 0 \\
\hline Vomiting & $5(0.6)$ & $3(0.7)$ \\
\hline Cerebral malaria & 0 & $1(0.2)$ \\
\hline
\end{tabular}

* The events listed occurred in at least $2 \%$ of patients in either treatment group. $\uparrow$ Seizure and grand mal seizure were the only serious adverse events thought to be treatment-related.

IAdverse events leading to study withdrawal were the same as those leading to discontinuation of treatment.

tem and the partner drug to eradicate residual parasites. Mefloquine has a half-life of about 3 weeks, ${ }^{20,21}$ as compared with about 2 weeks for pyronaridine. Pailin is a low-transmission area for P. falciparum, so patient immunity is limited. Thus, a combination of artemisinin resistance, differ- 
ences in partner-drug pharmacokinetics, and patient immunity may have contributed to the higher recrudescence rate through day 42 in Cambodian patients treated with pyronaridineartesunate.

According to a recently published study, ${ }^{19}$ the risk of infection with P. vivax after treatment for infection with $P$. falciparum is reduced when the treatment includes slowly eliminated drugs, such as mefloquine, rather than more rapidly eliminated drugs. Our study supports these findings, given the higher postbaseline rate of infection with $P$. vivax among patients receiving pyronaridineartesunate as compared with those receiving mefloquine plus artesunate. Among the 83 cases of postbaseline infection with $P$. vivax noted in our study, 73 (86.9\%) occurred in Thailand, where it is the most frequent cause of parasitologic failure after treatment for P. falciparum infection and occurs more frequently than would be expected from simple transmission. ${ }^{19}$ Although the pharmacokinetic-pharmacodynamic profile for pyronaridine has not been sufficiently determined, clinical evidence suggests that effective concentrations are probably intermediate - maintained for a longer period than for lumefantrine, ${ }^{10}$ but not for as long as for mefloquine. Artemisininbased combination therapy, in which the partner drug has a long effective half-life, does provide post-treatment prophylaxis, extending the infection-free interval. However, debate regarding the possible increased potential for the development of resistance to drugs with long half-lives continues. ${ }^{19,22-24}$

In terms of safety, our findings were generally consistent with reports on pyronaridine and artesunate monotherapy in Africa and Thailand. ${ }^{25-28}$ Our results were also consistent with those of clinical trials in which the drugs were used in combination. ${ }^{9-11}$

Combining the results of our study and two other phase 3 studies, one concerning P. falciparum $^{10}$ and the other $P$. vivax, ${ }^{11}$ transient increases in serum levels of alanine aminotransferase to more than five times the upper limit of the normal range occurred in 30 of 1925 patients (1.6\%). In these combined studies, 5 of the 1925 patients $(0.3 \%)$ had increases in levels of alanine aminotransferase that were more than three times the upper limit of the normal range and increases in total bilirubin that were more than two times the upper limit of the normal range. In 3 of these 5 patients, the guidelines on the use of Hy's law ${ }^{31}$ may apply. In the other 2 patients, additional factors may have contributed to the changes in liver function. Recovery typically occurred within a month, which is consistent with reversible liver injury. There were no other signs or symptoms of liver injury in any of these patients. In all the pyronaridine-artesunate studies conducted as part of the clinical development program to date, there have been no cases of liver failure.

Two patients receiving mefloquine plus artesunate had a seizure (one patient on day 2 and the other on day 3). The timing of these events suggests that they may have been caused by mefloquine. There was no known history of epilepsy, neurologic disorder, or concomitant epileptogenic drug use in either case. Seizures or convulsions are known to occur in response to mefloquine, usually after prophylaxis. ${ }^{29,30}$

Pyronaridine-artesunate has potential for the treatment of $P$. falciparum malaria., ${ }^{9}, 10$ After treatment with pyronaridine-artesunate, by day 28 , more than $95 \%$ of the patients at each of the Asian and African centers had an adequate clinical and parasitologic response, corrected for reinfection with the use of PCR genotyping. Although extended parasite clearance times in Cambodia were indicative of artemisinin resistance, artemisinin-based combination therapies remain important for the treatment of malaria.

\footnotetext{
Supported by a private-public partnership between Shin Poong Pharmaceutical Company and Medicines for Malaria Venture.

No potential conflict of interest relevant to this article was reported.

Disclosure forms provided by the authors are available with the full text of this article at NEJM.org.

We thank the study population and the study-center staff, all of whom made this study possible; Pongphaya Choosakulchart, Ghiorghis Belai, and David Asante (Family Health International), who were in charge of monitoring the study; Sarah Arbe-Barnes, Eric Didillon, Audrey Mulder (Fulcrum Pharma), and Claude Oeuvray (Medicines for Malaria Venture) for their dedicated work in the organization and execution of this trial; Hanspeter Marti and Hans-Peter Beck (Swiss Tropical and Public Health Institute), who performed central reading of the blood smears and the PCR analysis; Stephen Allen (Swansea University), Robert Miller (Fulcrum Pharma), J. Carl Craft (Libertyville, IL), and Frank Stephen Wignall (Family Health International) for their participation on the safety monitoring board; Steve Allen, Paul Watkins, Dominique Larrey, Tim Peto, Kwan Sik Lee, and Duolao Wang for their participation on the independent safety monitoring board examining hepatic safety; Mikaël Saulay and Arnaud Demange (Averion International), who performed the statistical analysis; and Naomi Richardson (Magenta Communications) for developing the first draft of the manuscript and for editorial assistance. Fulcrum Pharma and Averion International are now part of Aptiv Solutions.
} 
REFERENCES

1. Guidelines for the treatment of malaria. 2nd ed. Geneva: World Health Organization, 2010 (http://whqlibdoc.who .int/publications/2010/9789241547925_eng .pdf).

2. Maude RJ, Pontavornpinyo W, Saralamba $S$, et al. The last man standing is the most resistant: eliminating artemisininresistant malaria in Cambodia. Malar J 2009;8:31.

3. Containment of malaria multi-drug resistance on the Cambodia-Thailand border: report of an informal consultation Phnom Penh, 29-30 January 2007. Geneva: World Health Organization, 2007 (http:/l www.who.int/malaria/publications/ multi_drug_resistance_en.pdf).

4. Global malaria control and elimination: report of a meeting on containment of artemisinin tolerance. Geneva: World Health Organization, 2008 (http:/ whqlibdoc.who.int/publications/2008/ 9789241596817_eng.pdf).

5. Guerin PJ, Bates SJ, Sibley CH. Global resistance surveillance: ensuring antimalarial efficacy in the future. Curr Opin Infect Dis 2009;22:593-600.

6. Dondorp AM, Nosten F, Yi P, et al. Artemisinin resistance in Plasmodium falciparum malaria. N Engl J Med 2009;361:455-67. [Erratum, N Engl J Med 2009;361:1714.

7. Delacollette C, D'Souza C, Christophel $\mathrm{E}$, et al. Malaria trends and challenges in the Greater Mekong Subregion. Southeast Asian J Trop Med Public Health 2009;40: 674-91.

8. Wongsrichanalai C, Meshnick SR. Declining artesunate-mefloquine efficacy against falciparum malaria on the Cambodia-Thailand border. Emerg Infect Dis 2008;14:716-9.

9. Ramharter M, Kurth F, Schreier AC, et al. Fixed-dose pyronaridine-artesunate combination for treatment of uncomplicated falciparum malaria in pediatric patients in Gabon. J Infect Dis 2008;198:911-9.

10. Tshefu AK, Gaye O, Kayentao K, et al. Efficacy and safety of a fixed-dose ora combination of pyronaridine-artesunate compared with artemether-lumefantrine in children and adults with uncomplicat- ed Plasmodium falciparum malaria: a randomised non-inferiority trial. Lancet 2010; 375:1457-67.

11. Poravuth Y, Socheat D, Rueangweerayut $\mathrm{R}$, et al. Pyronaridine-artesunate versus chloroquine in patients with acute Plasmodium vivax malaria: a randomized, doubleblind, non-inferiority trial. PLoS One 2011; 6(1):e14501.

12. Assessment and monitoring of antimalarial drug efficacy for the treatment of uncomplicated falciparum malaria. Geneva: World Health Organization, 2003 (http://whqlibdoc.who.int/hq/2003/ WHO_HTM_RBM_2003.50.pdf)

13. Severe falciparum malaria. Trans $R$ Soc Trop Med Hyg 2000;94:Suppl 1:S1-S90.

14. Felger I, Beck HP. Genotyping of Plasmodium falciparum: PCR-RFLP analysis. Methods Mol Med 2002;72:117-29.

15. Methods and techniques for clinical trials on antimalarial drug efficacy: genotyping to identify parasite populations. Geneva: World Health Organization, 2008 (http://whqlibdoc.who.int/publications/ 2008/9789241596305_eng.pdf).

16. Wongsrichanalai C, Wimonwattrawatee $\mathrm{T}$, Sookto $\mathrm{P}$, et al. In vitro sensitivity of Plasmodium falciparum to artesunate in Thailand. Bull World Health Organ 1999; 77:392-8.

17. Jambou R, Legrand E, Niang M, et al. Resistance of Plasmodium falciparum field isolates to in-vitro artemether and point mutations of the SERCA-type PfATPase6. Lancet 2005;366:1960-3.

18. Noedl H, Se Y, Schaecher K, Smith BL, Socheat D, Fukuda MM. Evidence of artemisinin-resistant malaria in western Cambodia. N Engl J Med 2008;359:2619-20.

19. Douglas NM, Nosten F, Ashley EA, et al. Plasmodium vivax recurrence following falciparum and mixed species malaria: risk factors and effect of antimalarial kinetics. Clin Infect Dis 2011;52:612-20. 20. Simpson JA, Watkins ER, Price RN, Aarons L, Kyle DE, White NJ. Mefloquine pharmacokinetic-pharmacodynamic models: implications for dosing and resistance. Antimicrob Agents Chemother 2000;44: 3414-24.
21. White NJ. Antimalarial pharmacokinetics and treatment regimens. Br J Clin Pharmacol 1992;34:1-10.

22. Douglas NM, Anstey NM, Angus BJ, Nosten F, Price RN. Artemisinin combination therapy for vivax malaria. Lancet Infect Dis 2010;10:405-16.

23. Stepniewska K, White NJ. Pharmacokinetic determinants of the window of selection for antimalarial drug resistance. Antimicrob Agents Chemother 2008;52: 1589-96.

24. Sinclair D, Zani B, Donegan S, Olliaro P, Garner P. Artemisinin-based combination therapy for treating uncomplicated malaria. Cochrane Database Syst Rev 2009;3:CD007483.

25. Price R, van Vugt $M$, Phaipun L, et al. Adverse effects in patients with acute falciparum malaria treated with artemisinin derivatives. Am J Trop Med Hyg 1999; 60:547-55.

26. Ribeiro IR, Olliaro P. Safety of artemisinin and its derivatives: a review of published and unpublished clinical trials. Med Trop (Mars) 1998;58:Suppl:50-3.

27. Ringwald P, Bickii J, Basco L. Randomised trial of pyronaridine versus chloroquine for acute uncomplicated falciparum malaria in Africa. Lancet 1996;347:24-8.

28. Ringwald P, Bickii J, Basco LK. Efficacy of oral pyronaridine for the treatment of acute uncomplicated falciparum malaria in African children. Clin Infect Dis $1998 ; 26: 946-53$.

29. Nevin RL. Epileptogenic potential of mefloquine chemoprophylaxis: a pathogenic hypothesis. Malar J 2009;8:188.

30. Bem JL, Kerr L, Stuerchler D. Mefloquine prophylaxis: an overview of spontaneous reports of severe psychiatric reactions and convulsions. J Trop Med Hyg 1992;95:167-79.

31. Guidance for industry [on] druginduced liver injury: premarketing clinical evaluation. Washington, DC: Food and Drug Administration, July 2009 (http:/l www.fda.gov/downloads/Drugs/Guidance ComplianceRegulatoryInformation/ Guidances/UCM174090.pdf).

Copyright (c) 2012 Massachusetts Medical Society. 